\title{
La importancia del bosque en la recarga hídrica natural del acuífero noreste de la ciudad de Guatemala
}

\author{
Isaac R. Herrera Ibáñez* \\ Instituto de Investigaciones Agronómicas y Ambientales, Facultad de Agronomía, \\ Universidad de San Carlos de Guatemala, Guatemala
}

*Autor al que se dirige la correspondencia: iherrerai@hotmail.com

Recibido: 01 de octubre 2015 / Revisión: 07 de abril 2016 / Aceptado: 30 de abril 2016 / Disponible en línea: 01 de agosto 2016

\section{Resumen}

Zn la región volcánica con superficie boscosa la recarga hídrica es importante, y este trabajo pretende realizar - un análisis sobre el cálculo de la recarga en estas áreas. La investigación fue ejecutada en la cuenca del río Los Ocotes en la ciudad de Guatemala y cubre una superficie de $63.6 \mathrm{~km}^{2}$. En este trabajo se realizó un balance hídrico del suelo para estimar la recarga hídrica al acuífero. Existen un conjunto de procedimientos para estimar la distribución entre las entradas como lluvia y las salidas a través de la evapotranspiración, el escurrimiento superficial y la recarga subterránea. Durante los años 2009 y 2010, se hicieron registros meteorológicos y se definieron áreas con distinto uso de la tierra, lo cual se integró a un sistema de información geográfica (SIG) y se comparó con el mapa geomorfológico a escala 1:50,000 para elaborar el mapa de recarga de la cuenca con los datos calculados. Los sectores cultivados mostraron una menor infiltración y por lo tanto una menor recarga que en los sectores donde el bosque se encuentra en estado natural. La protección de las zonas de bosque como principal área de aporte a las reservas de agua subterránea, son fundamentales para los procesos de gestión y planificación territorial.

Palabras claves: Balance, suelo, lluvia, evapotranspiración, infiltración

\begin{abstract}
Tn the volcanic region with forest area hydric recharge is important, and paper presents an analysis of the recharge evaluation applied to these areas. The research was conducted in Los Ocotes river basin in Guatemala City and extends over a surface of $63.6 \mathrm{~km}^{2}$. In this work, an hydric balance in the soil was realized for estimated the aquifer recharge. There is a set of procedures aimed at estimating the distribution between inputs or rain and outputs through evapotranspiration, surface run of and groundwater recharge. In 2009 and 2010 were carried meteorological records and areas with different territorial occupation have been defined, this was integrated to Geographical Information System (GIS) and the geomorphic map was compared to 1:50,000 scale, for the recharge map of the basin was drawn with the data estimate. The cultured sectors showed a smaller infiltration and therefore a smaller recharge than in the sectors where the wood is in its natural state. The protection of the areas of natural wood a main contributing area to the ground water reserves represents a fundamental aspect for the management processes and territorial planning.
\end{abstract}

Keywords: Balance, soil, rain, evapotranspiration, infiltration 


\section{Introducción}

El bosque y sus componentes dominantes, los árboles, sirven a muchos propósitos que pueden ser divididos básicamente por su papel productivo y su papel de servicio. Tradicionalmente el hombre ha visto al bosque principalmente por su faceta productiva como un recurso renovable, ya que el bosque provee materiales de construcción como madera, energéticos en forma de leña o carbón y pulpa para papel.

Un servicio ambiental del bosque es la captación y almacenamiento de agua de lluvia, que origina áreas de recarga hídrica. Esto hace que sea de suma importancia delimitar las áreas de recarga y cuantificar las láminas y volúmenes de agua a nivel de cuenca, que fueron los objetivos centrales de esta investigación.

La Food and Agriculture Organization of the United Nations (FAO), recalca que la humanidad se ha dado cuenta que el bosque juega un papel importante en el medio ambiente, ya que es el mayor sistema de intercambio de dióxido de carbono y oxígeno, e influye favorablemente en la recarga hídrica de los acuíferos (FAO, 2009).

En Guatemala es de interés social y para el país mismo, la reforestación y conservación de los bosques con el establecimiento y mantenimiento de plantaciones forestales, que ha logrado impactos positivos en la protección de bosques y fuentes de agua (Castro, 2011).

Durante las últimas décadas la ciudad de Guatemala ha crecido alarmantemente, como consecuencia de la migración de la población del interior del país a la ciudad y del crecimiento demográfico, existiendo más de dos millones de habitantes (Instituto Nacional de Estadística, 2002). Esto ha dado como resultado que las aguas superficiales de los ríos Las Vacas y Villalobos, transporten aguas residuales provenientes de la ciudad de Guatemala, que las hace inadecuadas para el consumo humano (Fajardo, 2006). Por lo descrito anteriormente, se ha incrementado la explotación de las aguas subterráneas, haciéndose necesario delimitar las áreas de recarga hídrica que surten al acuífero para su protección y manejo correcto.

Los acuíferos son sistemas físicos que poseen un funcionamiento regulado por la recarga, movimiento del agua, descarga y extracciones, siendo la característica de la recarga hídrica al acuífero la más difícil de cuantificar de manera confiable (Escuder et al., 2009). Además, a pesar de los avances significativos que se han obtenido en la determinación de áreas de recarga hídrica a nivel mundial, en Guatemala la forma de es- timación es por balances hidrológicos generales a nivel de cuenca hidrográfica, el análisis de fluctuaciones de los niveles freáticos, medidas de pérdidas de caudal en cursos influentes y manantiales, y métodos empíricos. Los métodos difieren en los volúmenes de recarga estimados, por la adopción de modelos conceptuales incorrectos y los errores derivados de las mediciones y de cálculo.

A pesar que los procesos y mecanismos de la recarga a los acuíferos son relativamente bien conocidos, la obtención de una estimación segura de la magnitud de la recarga natural presenta aún grandes dificultades. Ellas radican en la variabilidad espacial y temporal de las condiciones climatológicas, geomorfológicas y especialmente de la variabilidad espacial de los factores que determinan la ocurrencia y magnitud de la recarga, como la zona no saturada o clase de suelo, pendiente, tipo de cobertura vegetal y la humedad antecedente del medio.

La problemática que motivó esta investigación, partió del hecho que en la ciudad de Guatemala muchas zonas boscosas se han reducido y gran parte de la superficie se ha impermeabilizado, reduciendo las áreas de recarga hídrica natural. Sin embargo, la Empresa Municipal de Agua (Empagua), menciona que la subcuenca del río Los Ocotes es un área potencial para el aprovechamiento del agua subterránea para la capital, con buena cobertura forestal que garantiza el proceso de infiltración de la lluvia, para que el almacenamiento del acuífero se mantenga en equilibrio y la explotación del agua sea sostenible (Empagua, 1990).

La caracterización hidrogeológica de la subcuenca del río Los Ocotes, identifica un acuífero volcánico en tobas fracturadas, cuyo espesor es superior a los $200 \mathrm{~m}$ (Herrera, 2012). El acuífero es semiconfinado, con permeabilidad media y abastece de agua potable a las zonas 15, 16, 17, 18 y 24, así como, a parte de San José Pinula y Santa Catarina Pinula, por lo que fue importante definir correctamente las áreas de recarga hídrica para su protección y manejo por parte de las municipalidades involucradas.

La subcuenca tiene un área de $63.6 \mathrm{~km}^{2}$ y se localiza en las coordenadas geográficas de $14^{\circ} 31^{\prime} 53^{\prime \prime}$ ' a $14^{\circ} 39^{\prime} 25^{\prime \prime}$ de latitud Norte y $90^{\circ} 24^{\prime} 02^{\prime \prime}$ a $90^{\circ} 28^{\prime} 37^{\prime \prime}$ de longitud Oeste. 


\section{Materiales y métodos}

La metodología utilizada fue la desarrollada por Herrera y Brown (2011), que se divide en tres fases: la primera comprende la obtención del mapa de unidades de recarga hídrica para el muestreo de campo, de acuerdo al traslape de los mapas de geomorfología y de uso actual de la tierra. En una segunda fase se realiza el cálculo de la recarga hídrica de cada unidad obtenida, por medio de los datos de clima y de los parámetros físicos del suelo, de acuerdo a la ecuación del balance: Precipitación $=$ Evapotranspiración + Escurrimiento + Retención + Recarga potencial.

Después de separar las pérdidas por escurrimiento superficial y retención en bosques y cultivos, el cálculo de recarga potencial al acuífero (Rp) se determina como: $\mathrm{Rp}=\mathrm{Pi}+\mathrm{Hsi}+\mathrm{Hsf}+\mathrm{ETR}$.

Donde Pi es la precipitación mensual que infiltra. Hs es la humedad del suelo, que es inicial (i) y final (f). La ETR es la evapotranspiración real (Schosinsky, 2006).

En la tercera fase las unidades cuantificadas se agrupan en un mapa final de recarga hídrica de acuerdo a los rangos de volumen de recarga específica anual, como muy altas con volúmenes mayores de 300,000 $\mathrm{m}^{3} / \mathrm{km}^{2} / \mathrm{anno}$; altas entre 150,000 a $300,000 \mathrm{~m}^{3} / \mathrm{km}^{2} /$ año; medias con recargas de 50,000 a $150,000 \mathrm{~m}^{3} / \mathrm{km}^{2} /$ año y áreas con recarga baja con volúmenes menores de $50,000 \mathrm{~m}^{3} / \mathrm{km}^{2} /$ año (Herrera, 2005).

Para la subcuenca del río Los Ocotes, el mapa geomorfológico se elaboró mediante la fotointerpretación de fotografías aéreas a escala 1:40,000, el cual se validó en campo. La geomorfología comprendió definir las unidades con una misma litología, similar topografía en función de la pendiente y las características físicas del suelo: textura y profundidad efectiva. El mapa final se elaboró a escala 1:50,000.

La cobertura vegetal fue determinada con ortofotos a escala 1:10,000 del año 2006, con la posterior comprobación de campo. La unidad mínima de mapeo considerada fue 6.25 ha.

La generación de las unidades de recarga hídrica natural, se realizó por medio de la sobreposición de las capas temáticas a escala 1:50,000 de geomorfología y uso actual de la tierra. Los mapas se elaboraron con sistemas de información geográfica, utilizando MapInfo ${ }^{\circledR}$ para dar solución al problema de utilización de bases cartográficas a diferentes escalas. Con base en estas unidades se realizaron las pruebas de infiltración con el método de Porchet (Sanders, 1998) y el muestreo de suelos a nivel de campo, con la posterior determinación en laboratorio de la capacidad de campo, punto de marchitez y densidad aparente.

Desde el punto de vista de la meteorología, se definió la precipitación pluvial como la principal variable de la recarga de los acuíferos y la evapotranspiración como pérdida en el sistema hídrico. El método utilizado para determinar la evapotranspiración potencial fue el de Hargreaves (Herrera, 2012).

Los parámetros meteorológicos se analizaron de forma diaria y mensual de los años 2009 y 2010, por medio de la instalación de dos estaciones climáticas tipo $\mathrm{C}$ o de tercer orden, que midieron precipitación pluvial, temperatura y humedad relativa. Una estación se ubicó en la parte alta de la subcuenca, en la aldea Ciénaga Grande en San José Pinula a una elevación de 1,915 msnm y la otra estación El Maestro en Lomas del Norte zona 17, en la parte baja de la subcuenca a 1,552 msnm (Herrera, 2012).

Posteriormente se hizo el calculó de láminas y volúmenes de recarga, se construyó el mapa de áreas de recarga hídrica anual de la subcuenca.

\section{Resultados}

\section{Geomorfología}

En la subcuenca del río Los Ocotes se distinguen tres unidades geomorfológicas (Figura 1): montañas volcánicas, relleno piroclástico y valle tectónico de la ciudad de Guatemala.

La unidad de montañas volcánicas se localiza en el centro de la subcuenca y representa el $40 \%$ del área. Se encuentra constituida por rocas volcánicas terciarias como tobas, basaltos y andesitas. La geomorfología típica corresponde a colinas y laderas, con pendientes entre 30 a $60 \%$. Los suelos son de la serie Morán, de texturas francos arenosos, profundos y bien drenados. Las elevaciones varían de los 1,300 a 1,900 msnm (Herrera, 2012).

La unidad de relleno piroclástico se ubica en las partes norte y sur, caracterizándose por presentar suelos de la serie Guatemala, francos arcillosos, profundos, mal drenados y desarrollados a partir de ceniza volcánica débilmente cementada sobre piroclastos de pómez. Las pendientes van de 5 a $10 \%$ en las partes onduladas y hasta de $100 \%$ en los taludes de los ríos. Las elevaciones son por lo general de 1,400 a 1,500 msnm.

La tercera unidad corresponde a un graben o depresión tectónica, limitado por los pilares tectónicos de Mixco al oeste y Santa Catarina Pinula al este, constituyendo el 
valle geotectónico de la ciudad de Guatemala. De acuerdo al Instituto Nacional de Sismología, Vulcanología, Meteorología e Hidrología (Insivumeh), los sistemas de fallas de Mixco y Santa Catarina Pinula, son fallas normales y con orientación de norte a sur, fácilmen- te identificables por sus escarpes de falla (Insivumeh, 1978). Esta unidad está constituida por piroclastos de pómez, con suelos de la serie Guatemala, francos arcillosos y profundos. Las pendientes van de 18 a $80 \%$ y las elevaciones de 1,500 a 1,550 msnm.

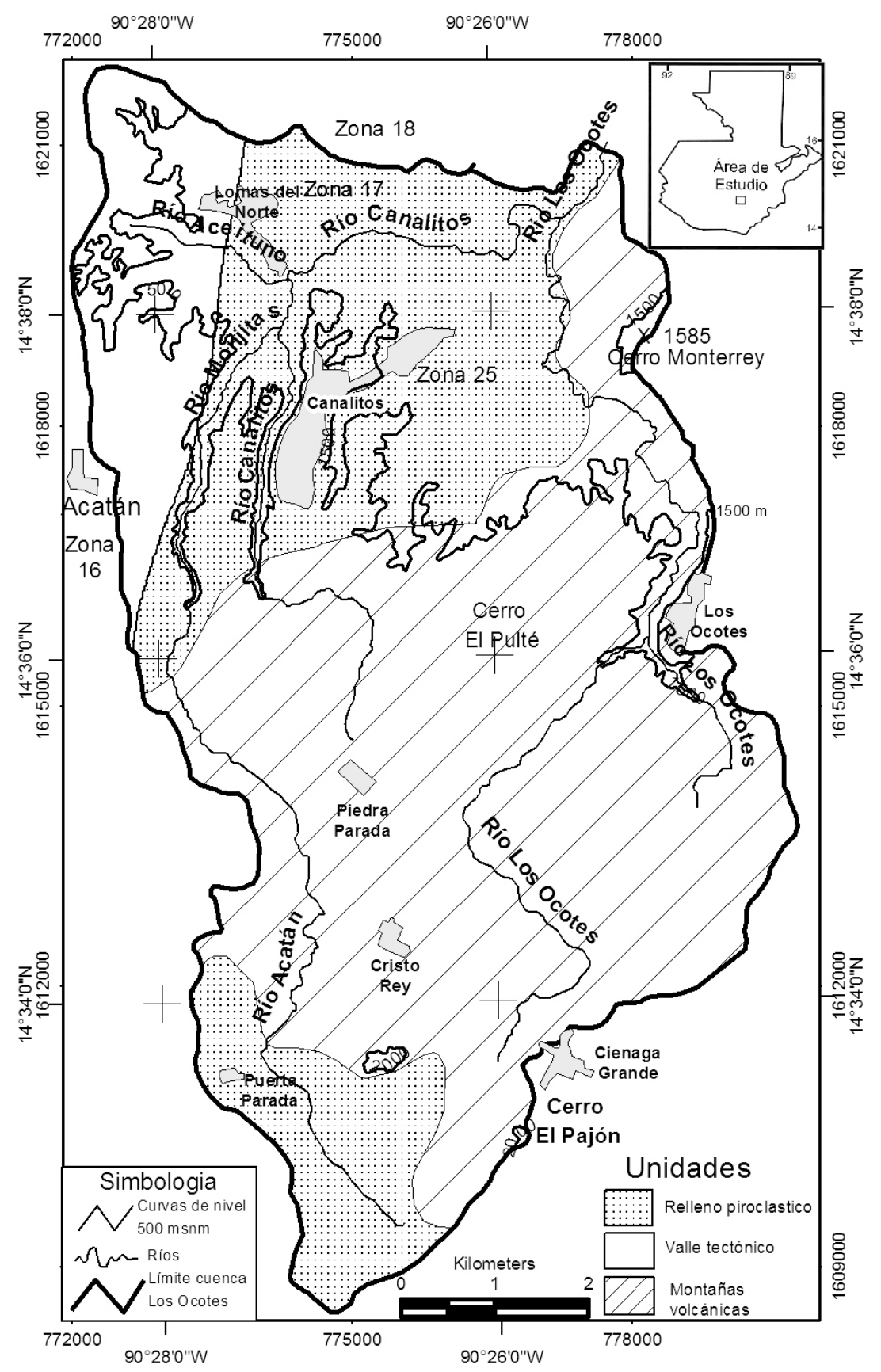

Figura 1. Geomorfología de la subcuenca del río Los Ocotes. 


\section{Uso actual de la tierra}

En la subcuenca del río Los Ocotes, predomina la cobertura de bosque de coníferas y encino (bosque mixto), como se observa en la Figura 2, con un área de $38.9 \mathrm{~km}^{2}$ que corresponde a un $61.16 \%$, seguido por el área con cultivos anuales de maíz y frijol, así como, café bajo sombra con un área de $11.26 \mathrm{~km}^{2}$ que representa el
$17.70 \%$ y áreas pequeñas con pasto de $1.33 \mathrm{~km}^{2}$ equivalente a $2.09 \%$. El resto de la superficie de la subcuenca de $12.11 \mathrm{~km}^{2}$ ó $19.05 \%$, comprende las áreas urbanizadas donde se asientan las poblaciones de Santa Lucía Los Ocotes, Canalitos, Lomas del Norte y Acatán del municipio de Guatemala. Así como, Piedra Parada, Cristo Rey y Puerta Parada en Santa Catarina Pinula y Ciénaga Grande en San José Pinula.

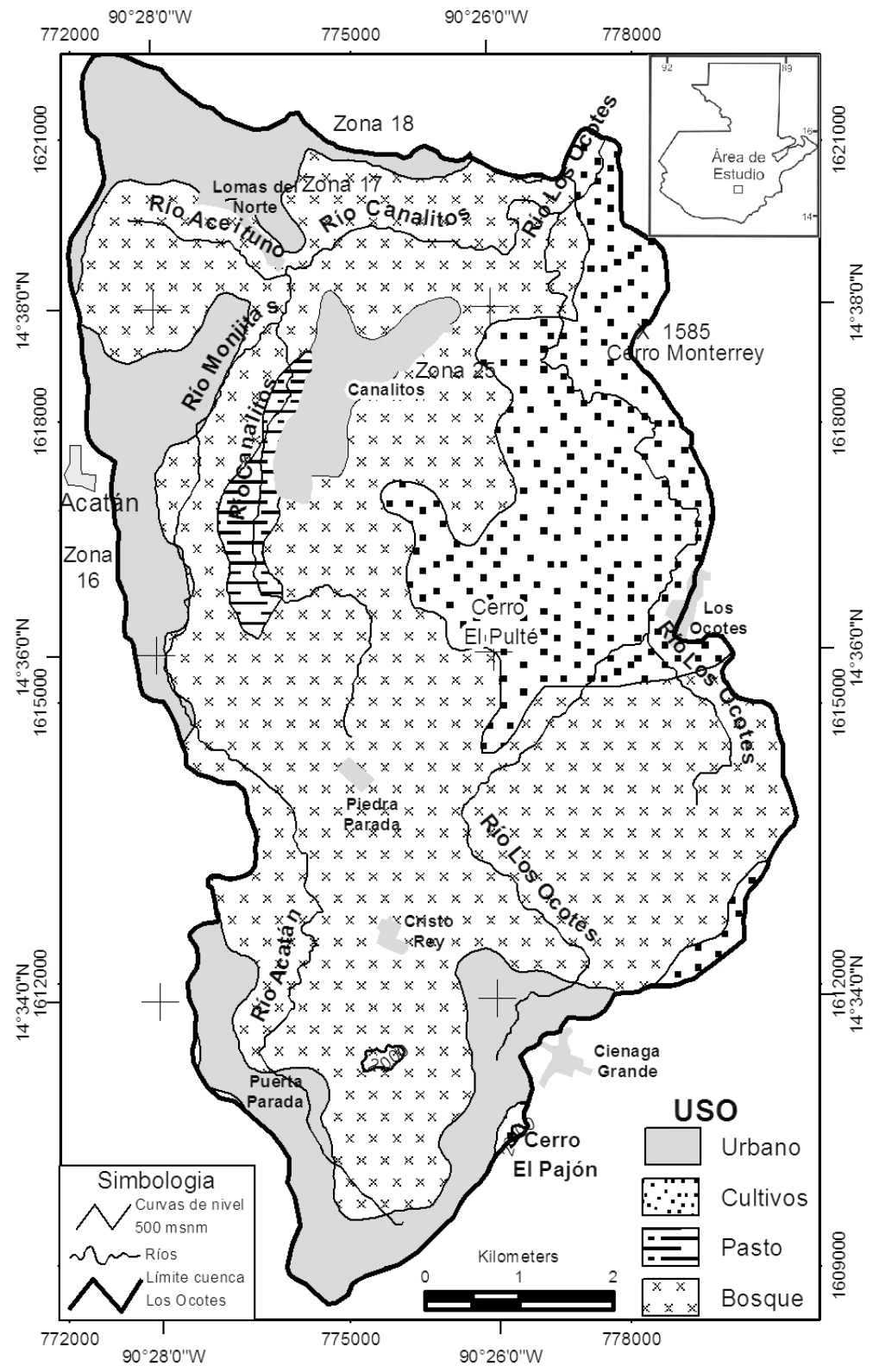

Figura 2. Uso actual de la tierra de la subcuenca del río Los Ocotes. 


\section{Clima}

La estación climática Ciénaga Grande representa el clima de la parte alta de la subcuenca del río Los Ocotes para el periodo 2009 y 2010 (Tabla 1).

La precipitación anual es de 1,386.3 $\mathrm{mm}$. Los meses que representan la época lluviosa con más de $200 \mathrm{~mm}$ por mes son: mayo, junio, julio, agosto y septiembre. Mientras que los meses secos son enero, febrero y marzo. La temperatura promedio es $18.2^{\circ} \mathrm{C}$. La humedad relativa media mensual es $51.9 \%$. La evapotranspiración presenta valores mensuales entre 119.3 a $168 \mathrm{~mm}$, con un valor anual de $1,724.2 \mathrm{~mm}$.

La estación climática El Maestro en la zona 17, representa el clima de la parte baja de la subcuenca del río Los Ocotes (Tabla 2). La precipitación media anual es de 1,318.4 mm. Los meses lluviosos son: mayo, junio, julio, agosto y septiembre. Los meses secos son: enero, febrero y marzo. Las temperaturas medias mensuales oscilan entre $22.2{ }^{\circ} \mathrm{C}$ a $24.3^{\circ} \mathrm{C}$ y la temperatura anual es $23.4^{\circ} \mathrm{C}$. La humedad relativa media anual es $44 \%$. La evapotranspiración potencial mensual varía entre $150.6 \mathrm{~mm}$ a $201.6 \mathrm{~mm}$, siendo el valor anual de $2,159.8 \mathrm{~mm}$.

\section{Recarga hídrica}

Se utilizaron diez unidades para el cálculo de recarga hídrica como se presentan en la Tabla 3. Las unidades 1, 2, 3 y 4 corresponden a las montañas volcánicas.

Tabla 1

Datos de clima de la estación Ciénaga Grande 2009 - 2010

\begin{tabular}{|c|c|c|c|c|c|c|c|c|c|c|c|c|c|}
\hline Parámetro & Ene. & Feb. & Mar. & Abr. & May. & Jun. & Jul. & Ago. & Sept. & Oct. & Nov. & Dic. & Anual \\
\hline $\begin{array}{l}\text { Precipitación } \\
\text { pluvial }(\mathrm{mm})\end{array}$ & 0 & 0 & 0 & 48 & 247 & 255.8 & 234.8 & 241.6 & 216.6 & 50.8 & 59.6 & 32.3 & $1,386.3$ \\
\hline $\begin{array}{l}\text { Temperatura } \\
\left({ }^{\circ} \mathrm{C}\right)\end{array}$ & 18.6 & 17.6 & 18.7 & 20.6 & 16.5 & 16.0 & 16.6 & 19.0 & 19.2 & 19.5 & 18.7 & 17.8 & 18.2 \\
\hline $\begin{array}{l}\text { Humedad } \\
\text { relativa }(\%)\end{array}$ & 54.6 & 54.4 & 49.9 & 51.3 & 55.2 & 55.5 & 54.7 & 46.9 & 47.3 & 52.1 & 50.6 & 50.3 & 51.9 \\
\hline $\mathrm{ETP}(\mathrm{mm})$ & 123.4 & 119.3 & 152.9 & 164.3 & 151.2 & 143.6 & 151.8 & 168.0 & 157.2 & 146.7 & 126.1 & 119.6 & $1,724.2$ \\
\hline
\end{tabular}

Nota. ETP $=$ Evapotranspiración potencial

Tabla 2

Datos de clima estación El Maestro 2009 - 2010

\begin{tabular}{|c|c|c|c|c|c|c|c|c|c|c|c|c|c|}
\hline Parámetro & Ene. & Feb. & Mar. & Abr. & May. & Jun. & Jul. & Ago. & Sept. & Oct. & Nov. & Dic. & Anual \\
\hline $\begin{array}{l}\text { Precipitación } \\
\text { pluvial (mm) }\end{array}$ & 0.8 & 0 & 0 & 68.3 & 209.9 & 286.6 & 173.1 & 309.2 & 173.6 & 19.5 & 38.5 & 39.1 & $1,318.4$ \\
\hline $\begin{array}{c}\text { Temperatura } \\
\left({ }^{\circ} \mathrm{C}\right)\end{array}$ & 22.2 & 23.5 & 23.8 & 23.5 & 24.3 & 23.8 & 24.0 & 23.1 & 23.8 & 23.0 & 22.5 & 22.9 & 23.4 \\
\hline $\begin{array}{l}\text { Humedad } \\
\text { relativa }(\%)\end{array}$ & 41.2 & 46.0 & 40.0 & 35.6 & 46.4 & 48.0 & 48.3 & 49.6 & 48.0 & 42.9 & 42.6 & 39.6 & 44.0 \\
\hline ETP (mm) & 152.3 & 150.6 & 191.0 & 197.5 & 201.6 & 191.1 & 191.4 & 191.9 & 186.6 & 184.0 & 163.3 & 151.5 & $2,159.8$ \\
\hline
\end{tabular}

Nota. ETP = Evapotranspiración potencial 
Tabla 3

Recarga hídrica de la subcuenca del río Los Ocotes.

\begin{tabular}{lcccccc}
\hline No. & $\begin{array}{c}\text { Unidad } \\
\text { geomorfológica }\end{array}$ & Cobertura & $\begin{array}{c}\text { Lámina } \\
\text { de recarga } \\
(\mathrm{m})\end{array}$ & $\begin{array}{c}\text { Área } \\
\left(\mathrm{Km}^{2}\right)\end{array}$ & $\begin{array}{c}\text { Volumen de } \\
\text { recarga } \\
\left(\mathrm{x} 10^{6} \mathrm{~m}^{3}\right)\end{array}$ & $\begin{array}{c}\text { Recarga } \\
\text { específica }\left(\mathrm{m}^{3} /\right. \\
\left.\mathrm{km}^{2}\right)\end{array}$ \\
\hline 1 & Montañas Volcánicas & Bosque & 0.124 & 24.0812 & 2.995 & 124,390 \\
2 & Montañas Volcánicas & Cultivos & 0.069 & 9.6848 & 0.672 & 69,430 \\
3 & Montañas Volcánicas & Pastos & 0.074 & 0.4550 & 0.033 & 73,600 \\
4 & Montañas Volcánicas & Poblados & 0.00 & 1.83654 & 0.00 & 0 \\
5 & Relleno Piroclástico & Poblados & 0.00 & 6.3794 & 0.00 & 0 \\
6 & Relleno Piroclástico & Cultivos & 0.034 & 1.54605 & 0.053 & 34,170 \\
7 & Relleno Piroclástico & Pastos & 0.044 & 0.8910 & 0.039 & 44,290 \\
8 & Relleno Piroclástico & Bosque & 0.031 & 12.3345 & 0.386 & 31,320 \\
9 & Valle Tectónico & Poblados & 0.00 & 3.9950 & 0.00 & 0 \\
10 & Valle Tectónico & Bosque & 0.00 & 2.3986 & 0.00 & 0 \\
& Total $=$ & & & 63.6021 & 4.18 & 0 \\
\hline
\end{tabular}

Mientras que las unidades 5, 6, 7 y 8 , al relleno piroclástico, y las restantes 9 y 10 pertenecen al valle tectónico.

Los datos de lluvia y evapotranspiración de la estación Ciénaga Grande, se tomaron para las unidades de la parte alta y media de la subcuenca, mientras que para el cálculo de las unidades de la parte baja, se tomaron los datos de la estación El Maestro.

La recarga hídrica al acuífero varía de 31 a 124 $\mathrm{mm} / \mathrm{año}$, encontrándose entre el rango de valores de 30 a $370 \mathrm{~mm} /$ año cuantificados para regiones tropicales con considerables variaciones anuales de lluvia (Carrica, 2009; Del Valle \& Varni, 2009; Herrera, 2011). El volumen total de recarga en la subcuenca del río Los Ocotes es de 4.18 millones de $\mathrm{m}^{3}$.

La categorización de los resultados basándose en el volumen de recarga hídrica específica anual de cada unidad, cuantifica a las unidades 1, 2 y 3, de montañas volcánicas con bosques, cultivos y pastos, como áreas con recarga entre 69,430 a $124,390 \mathrm{~m}^{3} / \mathrm{km}^{2}$, clasificándose como media y presentándose en los alrededores de Los Ocotes, Piedra Parada, Cristo Rey y Ciénaga Grande. Las unidades 6, 7 y 8 de relleno piroclástico con cultivos, pastos y bosques, se clasifican como áreas de baja recarga por presentar valores de recarga menores a 50,000 $\mathrm{m}^{3} / \mathrm{km}^{2}$, en los alrededores de Canalitos y Puerta Parada. Mientras que las restantes unidades 4, 5 , 9 y 10 , se calcularon valores de 0 y se clasifican como áreas sin recarga potencial, ya que son áreas muy poco permeables y donde se asientan las poblaciones de la subcuenca (Figura 3).

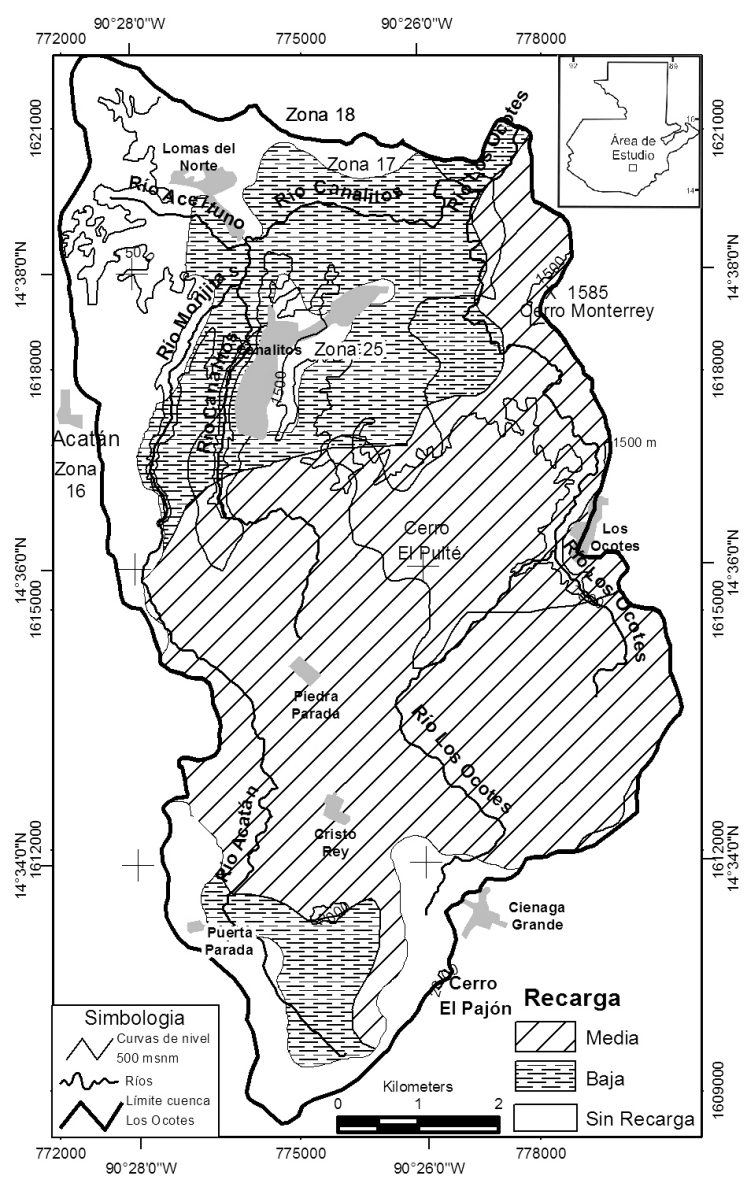

Figura 3. Áreas de recarga hídrica de la subcuenca del río Los Ocotes. 


\section{Discusión}

\section{Geomorfología}

La geomorfología distingue el tipo litológico para determinar si el medio permeable es poroso o fisurado. Además, distingue el relieve para precisar la pendiente del terreno y la clase de suelo, que es importante en los procesos de infiltración, el escurrimiento, la humedad del suelo y la recarga.

La recarga hídrica natural depende de la cantidad de agua absorbida a través del suelo y percolada en las rocas hasta llegar al acuífero. En los terrenos con pendientes suaves el agua de lluvia tiene más tiempo para poder ser infiltrada, resultando una mayor cantidad de agua almacenada en el suelo y la posibilidad de tener más recarga. Caso contrario ocurre en los terrenos con altas pendientes, donde el mayor porcentaje de agua de lluvia se escurre superficialmente.

La velocidad de infiltración del agua en los suelos es función de la textura. Esto se pudo constatar en la subcuenca del río Los Ocotes, donde las velocidades varían de $144 \mathrm{~mm} /$ día para suelos franco arcillosos hasta $960 \mathrm{~mm}$ /día para suelos franco arenosos. Las texturas arcillosas se encuentran en el valle tectónico de la subcuenca, ubicadas en superficies de poca infiltración y por ende de muy poca recarga o sin recarga hídrica potencial.

Las texturas franco arenosas corresponden a las áreas de montañas volcánicas con bosque, calculándose láminas de recarga de 124 mm/año. Mientras que en las áreas de relleno piroclástico con bosque, las láminas de recarga son más bajas de $31 \mathrm{~mm} / \mathrm{año}$. Una situación similar se presenta en la unidad de montañas volcánicas con pasto y cultivo, con láminas de recarga de 74 y $69 \mathrm{~mm} / \mathrm{año}$ respectivamente. Las láminas de recarga de esta cobertura también son menores en la unidad de relleno piroclástico, donde se obtuvieron valores de $44 \mathrm{~mm} /$ año para pasto y de $34 \mathrm{~mm} /$ año para cultivos.

Las profundidades de las raíces de $0.40 \mathrm{~m}$ para áreas con maíz, $0.20 \mathrm{~m}$ para áreas con pastos, mientras que las áreas con bosque tienen una profundidad de raíces de aproximadamente $1.5 \mathrm{~m}$.

\section{Bosque}

El agua que alcanza la superficie del suelo de un bosque por lluvia, tiene varias opciones para entrar a formar parte del ciclo hidrológico. Una parte puede permanecer en la superficie de donde un porcentaje se evapora directamente, otro pequeño porcentaje podría correr sobre la superficie como flujo superficial y el resto se infiltrará en el suelo.

El agua se almacenará en el suelo hasta un cierto nivel máximo que depende de las propiedades físicas del suelo y es principalmente de este "reservorio" que la vegetación forestal toma el agua requerida para la transpiración. La cantidad de agua que puede ser utilizada por los árboles depende de la fracción de agua aprovechable (diferencia entre el contenido actual de humedad y el contenido en el punto de marchites permanente) y la profundidad de la capa de suelo que puede ser aprovechada por el sistema de raíces. Si se infiltra más agua de la que el suelo puede contener, esta continuará percolándose hacia el agua subterránea, produciéndose la recarga hídrica al sistema acuífero.

En la subcuenca del río Los Ocotes las láminas infiltradas anuales para los años 2009 y 2010, son mayores en cobertura boscosa, con valores de 310 a $1,240 \mathrm{~mm}$, son medias en pasto con rangos de 440 a $740 \mathrm{~cm}$ y menores en cultivos con 340 a $690 \mathrm{~cm}$. De acuerdo a las recargas específicas, estas son mayores en bosques con 31,320 a $124,390 \mathrm{~m}^{3} / \mathrm{km}^{2}$, que en pastos con 44,290 a $73,600 \mathrm{~m}^{3} / \mathrm{km}^{2}$ y cultivos con 34,170 a $69,430 \mathrm{~m}^{3} / \mathrm{km}^{2}$. Además, como las lluvias en el trópico frecuentemente caen más rápidamente de lo que puede ser absorbidas por el suelo, es decir, la intensidad es mayor a la capacidad de infiltración, tal incremento de la capacidad de infiltración reducirá la sobrecarga y el flujo superficial en las áreas forestales.

Todos los componentes mencionados del ciclo del agua en el suelo, están influenciados por la vegetación forestal a través de su impacto en la infiltración y las propiedades físicas del suelo.

La capacidad de infiltración presenta una correlación positiva con la cobertura de árboles, lo que implica que la infiltración mejora donde hay una mayor cobertura arbórea (Casado, Gil, \& Campo, 2007).

La cama de suelo forestal protege efectivamente el suelo y previene la obstrucción de los espacios porosos que constituyen las vías para la infiltración del agua. Esto ocurre en menor grado en la cobertura de pastos y mucho menor donde se encuentran cultivos.

El sistema de raíces profundo de los árboles, el alto contenido de materia orgánica y la bien desarrollada actividad micro orgánica mejoran las propiedades del suelo e incrementan la capacidad de almacenamiento de agua de los suelos forestales, pero también contribuyen a una mayor detención temporal del almacenamiento (FAO, 2009). 
A mayor cantidad de agua retenida y disponible para el uso de la planta, menor será la contribución a la escorrentía y a la recarga del agua subterránea, y el aporte de agua de exceso a la escorrentía en la cuenca se retrasará.

\section{Clima}

La ciudad de Guatemala presenta un clima templado, con una precipitación pluvial anual mayor de $1,000 \mathrm{~mm}$. Esto se comprobó en las dos estaciones climáticas dentro de la subcuenca del río Los Ocotes para los años 2009 y 2010, donde en la parte alta la precipitación anual fue de 1,386.3 $\mathrm{mm}$ y en la parte baja la lluvia anual fue de $1,318.4 \mathrm{~mm}$, existiendo solamente una diferencia de $67.9 \mathrm{~mm}$ entre las dos estaciones. Además, la época lluviosa se presenta de mayo a septiembre, mientras que la época seca es de enero a marzo, siendo los meses de octubre, noviembre, diciembre y abril con poca lluvia.

La temperatura media de $18.2^{\circ} \mathrm{C}$ se presenta en la parte alta de la subcuenca con $1,915 \mathrm{msnm}$, mientras que en la parte baja a $1,552 \mathrm{msnm}$ es $23.4^{\circ} \mathrm{C}$, existiendo un gradiente de temperatura de $1.4^{\circ} \mathrm{C}$ por cada $100 \mathrm{~m}$.

En la parte alta de la subcuenca la evapotranspiración es menor y presenta un valor anual de 1,724.2 mm y en la parte baja el valor anual es mayor, con un valor de 2,159.8 mm. Esto es congruente con la transpiración del tipo de vegetación de bosques de coníferas que se presentan en la parte alta y media, y que tienen menores valores de transpiración de 15 a $39 \mathrm{~mm} / \mathrm{mes}$, mientras que el cultivo de maíz se presenta principalmente en la parte baja y tiene una mayor transpiración de 68 a 84 $\mathrm{mm} / \mathrm{mes}$ (Herrera, 2012).

Otro aspecto relevante de la investigación, es lo concerniente a que el bosque a veces no presenta recarga hídrica, ya sea porque se encuentra en suelos arcillosos donde existe una infiltración muy baja o porque la cantidad de lluvia es muy baja. Herrera, Orozco y Mujica (2011), definen que en las zonas donde la precipitación pluvial anual es menor de 1,000 mm, generalmente no existe recarga hídrica directa.

\section{Agradecimientos}

Este trabajo es parte de un proyecto de investigación financiado por el Fondo Nacional de Ciencia y Tecnología (FONACYT) del Consejo Nacional de Ciencia y Tecnología (CONCYT) con el número Fodecyt No. 35-2009 Estudio hidrogeológico de la subcuenca del río Los Ocotes, para determinar las áreas principales de recarga hídrica e identificación de las áreas vulnerables a deslizamientos e inundaciones para proponer alternativas de prevención de la parte noreste de la ciudad de Guatemala.

\section{Referencias}

Carrica, J. (2009). Cálculo de la recarga hídrica en zonas áridas y semiáridas. Trabajo presentado en el VI Congreso Nacional de Hidrogeología y IV Seminario hispanoamericano de Temas Actuales de la Hidrología Subterránea, Santa Rosa, La Pampa, Argentina.

Casado, A., Gil, V., \& Campo, A. (2007). Consecuencias de la variación de la disponibilidad hídrica en la cuenca del arroyo El Belisario, Buenos Aires, Argentina. Huellas, 11, 9-26.

Castro, M. (2011). Recarga hídrica natural en la subcuenca del río Tzalá del municipio de San Miguel Ixtahuacán, Sipacapa y Comitancillo del departamento de San Marcos, Guatemala (Tesis de licenciatura). Facultad de Agronomía, Universidad de San Carlos de Guatemala, Guatemala.

Del Valle, V., \& Varni, M. (2009). De los métodos específicos para cuantificar la recarga de acuíferos. Trabajo presentado en el VI Congreso Nacional de Hidrogeología y IV Seminario hispanoamericano de Temas Actuales de la Hidrología Subterránea, Santa Rosa, La Pampa, Argentina.

Empresa Municipal de Agua. (1990). Los recursos de aguas subterráneas para el abastecimiento a la ciudad de Guatemala. Guatemala: Autor.

Escuder, R., Fraile, J., Jordana, S., Ribera, F., Sánchez-Vila, X., \& Vázquez-Suñé, E. (2009). Hidrogeología, conceptos básicos de hidrología subterránea. Barcelona: Fundación Centro Internacional de Hidrología Subterránea.

Fajardo, R. (2006). Evaluación hidráulica mediante el uso de modelos del tramo final del río Villalobos (Tesis de maestría). Facultad de Ingeniería, Universidad de San Carlos de Guatemala, Guatemala.

Food and Agriculture Organization of the United Nations (2009). Regional study, practical experiences of compensation mechanisms for water services provided by forests in Central America and the Caribbean. Rome: Autor. 
Herrera, I. (2005). Estudio hidrogeológico de la parte norte de la caldera del lago de Atitlán, Guatemala. Trabajo presentado en el Congreso Internacional de Hidrogeología y Manejo de Recursos Hídricos. Managua, Nicaragua.

Herrera, I. (2011). Estrategia para el aprovechamiento sostenible del recurso hídrico subterráneo en la su-cuenca de los ríos Pansigüis y Cushapa, Departamento de Jalapa, Guatemala (Tesis de doctorado). Universidad Máximo Gómez Báez, Ciego de Ávila, Cuba.

Herrera, I. (2012). Estudio hidrogeológico de la subcuenca del río Los Ocotes, para determinar las áreas principales de recarga hídrica e identificación de las áreas vulnerables a deslizamientos e inundaciones para proponer alternativas de prevención de la parte noreste de la ciudad de Guatemala. (FODECYT No. 2009.35). Guatemala: Consejo Nacional de Ciencia y Tecnología, Universidad de San Carlos de Guatemala, Facultad de Agronomía.

Herrera, I., \& Brown, O. (2011). Propuesta de una metodología para la estimación de áreas de recarga hídrica en Guatemala. Revista Ciencias Técnicas Agropecuarias, 20(4) 48-52.
Herrera, I., Orozco, E., \& Mujica, A. (2011). Estrategia para el aprovechamiento del recurso hídrico subterráneo en una zona semiárida de Guatemala. Revista Ciencias Técnicas Agropecuarias, 20(3) 12-18.

Instituto Nacional de Estadística. (2002). Censos Nacionales, XI de Población y VI de Habitación. Guatemala: Autor.

Instituto Nacional de Sismología, Vulcanología, Meteorología e Hidrología (1978). Estudio de las aguas subterráneas en el valle de la ciudad de Guatemala. Guatemala: Autor.

Sanders, L. (1998). A manual of field hydrogeology. New Jersey: Prentice-Hall.

Schosinsky, G. (2006). Cálculo de recarga hídrica de acuíferos mediante balance hídrico de suelos. Revista Geológica de América Central, 34, 13-30. 